\title{
Antibody-drug conjugates: targeted weapons against cancer
}

This article was published in the following Dove Press journal:

Antibody Technology Journal

9 January 2015

Number of times this article has been viewed

\section{Luisa lamele \\ Luca Vecchia \\ Claudia Scotti}

Department of Molecular Medicine, Unit of Immunology and General Pathology, University of Pavia, Pavia, PV, Italy

All authors contributed equally to this work
Correspondence: Claudia Scotti, Department of Molecular Medicine, Unit of Immunology and General Pathology, University of Pavia, Via Ferrata 9, 27I00 Pavia, PV, Italy Tel +390382986335

$\mathrm{Fax}+390382986893$

Email claudia.scotti@unipv.it
Abstract: Antibody-drug conjugates (ADCs) are formed by a targeting antibody conjugated to a chemotherapeutic molecule through a linker. Recent data demonstrate that ADCs represent a valuable advancement for the clinics and, despite their recent appearance in medicine, they are already undergoing an innovation wave aimed at targeting all three ADC building blocks. Thus, new antibody formats are being engineered, site-specific linkers are being designed, and highly toxic molecules, like RNA polymerase inhibitors, are starting to be used for conjugation. These molecules were previously considered extremely toxic and could not be used as chemotherapeutic drugs. In this review, we will present an overview of current cancer treatments and their limitations, and the logic that has led to the generation of ADCs. Their mechanism of action will be outlined, and the most recent novelties in linker design and optimization will be discussed, along with present and near future discoveries in the current ADC research pipeline.

Keywords: immunoconjugates, antibody-drug conjugates, antibody fragments, cancer, monoclonal antibodies

\section{Introduction}

The amount of information and progress in the understanding of the cellular and molecular biology of cancer in recent years has given vast opportunities for future discoveries and the development of new diagnostic and therapeutic agents for the control of the disease. Cancer has long been treated with a variety of cytotoxic drugs with the aim of destroying the malignant cells without causing significant harm to the surrounding normal cells. ${ }^{1}$ In order to do that, many of these drugs preferentially kill cell types with higher proliferation or metabolic rates, a feature typical of most tumor cells but also of many healthy cells, such as some epithelia. Toxicity and side effects are therefore an unavoidable price to pay during chemotherapy treatment.

At present, cancer biologics (antibodies, peptides) and small molecules play an increasing role as therapeutic molecules, especially in combination with radiotherapy or chemotherapy. Antibody therapeutics have become fully established in the last decades, starting with the revolution of the hybridoma technology to develop murine monoclonal antibodies (MAbs). ${ }^{2}$ However, only 12 therapeutic MAbs are marketed today for the treatment of cancer, ${ }^{3}$ highlighting two main problems, the identification of the "perfect" molecular target, and the clinical efficacy of the MAb as a single therapeutic agent.

Among all antibody-related products, immunoconjugates have become increasingly important as oncology therapeutics. The concept of conjugated MAbs rises from the attempt to increase the specificity of chemo- or radiation therapy and 
improve the efficacy of immunotherapy by exploiting the specificity of the MAb for the target. The linked antibody in fact can significantly decrease non-specific uptake of the drug and increase specific uptake of the conjugate by the tumor cells. ${ }^{4}$ Immunoconjugates of various kinds constituted $44 \%$ of the total anticancer MAbs in clinical studies up to $2010 .{ }^{5}$

The main components of a therapeutic immunoconjugate are described in Figure 1. Immunoconjugates can be categorized into three groups depending on the nature of the molecule linked to the antibody. In the case of a protein toxin, the conjugate is an immunotoxin conjugate. In clinical studies, they have proven highly immunogenic and showed nonspecific toxicity, starting from damage to the endothelium and also hepatic and renal toxicity. ${ }^{6}$

When the conjugate carries an isotope as effector, it is referred to as a radioimmunoconjugate. Clinical studies have revealed the limitations of radioimmunoconjugates as cancer therapeutics, especially because of the difficulty of delivering effective radiation doses to solid tumors, the complex chemistry often required for conjugation, and the potentially toxic effects on normal tissues. ${ }^{7}$ The most clinically promising results have been obtained in treatment of hematologic cancers due to the radiosensitivity of these tumors and the ability to deliver more easily the necessary dose for tumor eradication. ${ }^{8}$

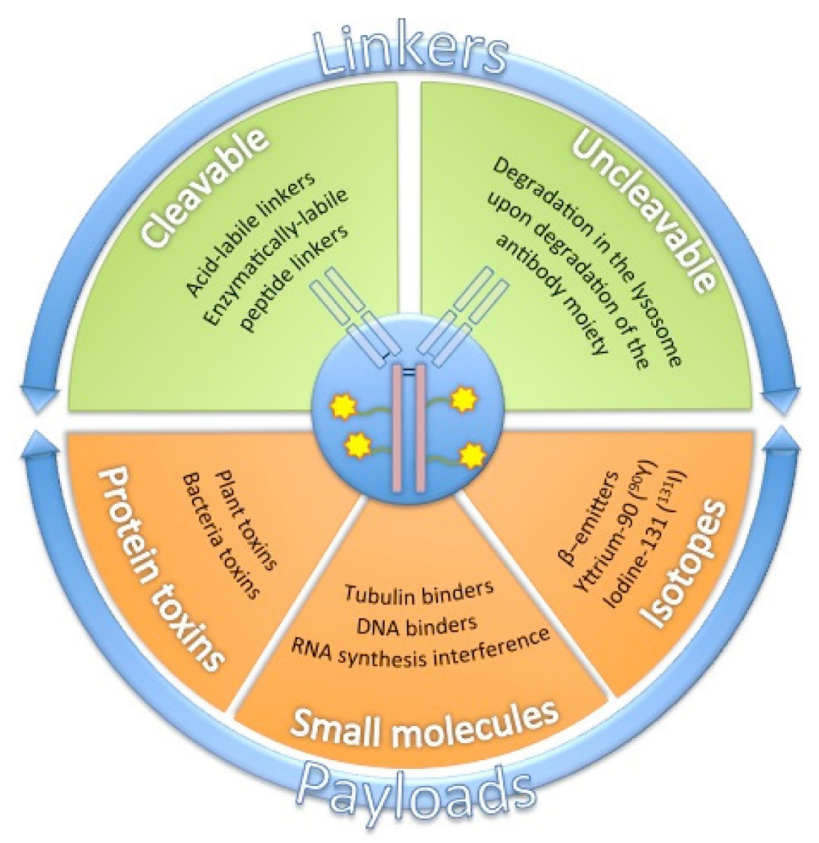

Figure I Immunoconjugate components. An antibody, shown in the center of the chart, is conjugated to an effector molecule (payload) belonging to one of three main categories (protein toxins, small molecules, or isotopes). The conjugation occurs via a linker, classified into two main categories (cleavable or uncleavable), depending on its chemical features.
The third group of immunoconjugates, which is also the main focus of this review, is formed by antibody-drug conjugates (ADCs), featuring antibodies armed with small drugs, generally of a molecular weight between 300 and 1,000 Da. The first ADC approved by the US FDA (Food and Drug Administration) in 2000 (but never in Europe) was gemtuzumab ozogamicin (Pfizer, Inc., New York, NY, USA), a calicheamicin-linked CD33-specific MAb for the treatment of acute myeloid leukaemia (AML). ${ }^{9}$ In 2010, however, the drug was withdrawn from the US market by the developing company after the failure of the required post-approval study. ${ }^{10}$

Gemtuzumab ozogamicin is considered a first-generation ADC that has opened the door to the improvement of the technology and has led to the design of new compounds, which are currently at different stages of clinical development. ${ }^{11}$

At the end of 2013, 35 novel ADCs were being investigated in clinical studies as treatments for a variety of solid and liquid tumors. Out of these 35 , nearly $70 \%$ entered clinical study in the previous 3 years. ${ }^{12}$

\section{ADCs: mechanism of action}

The basic concept in designing an ADC is to combine the extreme precision of the antibody directing the drug toward the cell surface together with the power of the cytotoxicity associated with the drug. ADCs are in this sense pro-drugs requiring drug release for activation, commonly after $\mathrm{ADC}$ internalization into the target cell. ${ }^{13}$ The mode of action of an $\mathrm{ADC}$ administered to a patient can be followed in the steps described in this paragraph and in Figure 2.

\section{Circulation}

The intravenously delivered ADC, while in circulation, must behave like a naked antibody in the plasma. In particular, the linker must be stable in the bloodstream, as decay would release the cytotoxin before being delivered to the target site and cause damage to the healthy tissues.

\section{Antigen binding}

The ADC binds to a target antigen on the surface of the tumor cell. The choice of the target is critical for the ADC to be a successful therapeutic. In the selection of a target, some essential features should generally be complied with that are similar to those applied in the design of naked therapeutic MAbs., ${ }^{3,14}$ However, the choice of a suitable target cannot be just as simple, and pros and cons of the main criteria considered will be described in the following paragraphs of this review. 


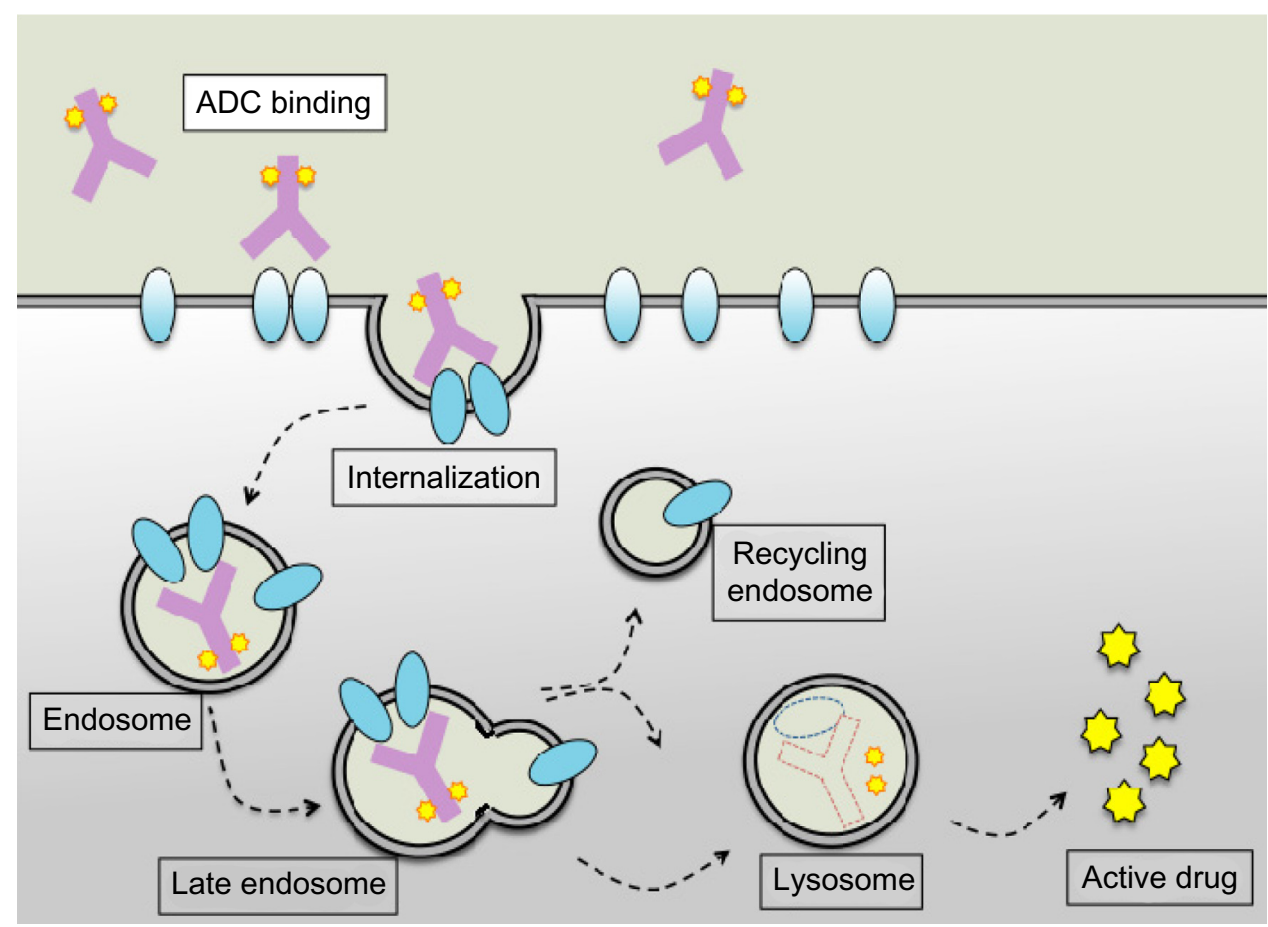

Figure 2 Mechanism of action of ADCs. The antibody-drug conjugate (purple $\mathrm{Y}$ shape with little stars) binds to the target receptor (blue ovals) on the cell surface and is internalized by endocytosis. Processing through the endosome-lysosome pathway leads to antibody detachment, recycling of the receptor toward the cell membrane and release of the effector molecule in the form of active drug.

Abbreviation: ADCs, antibody-drug conjugates.

\section{Internalization}

ADC internalization is generally necessary for efficient drug release in the cytoplasm, depending upon the drug and linker. The ideal scenario for a successful ADC would be that not only the target antigen is internalized, but also that a rapid internalization process, combined with efficient recycling of the antigen at the cell surface, should be desirable. In reality, none of these must be considered a fundamental criterion for target selection. There are examples of inefficiently internalising antigens that have been successfully targeted with ADCs,${ }^{15}$ as well as powerful ADCs with a slow internalising rate. ${ }^{16}$

\section{Drug release}

Once internalized, the ADC will move into the cells by an endocytosis process and through this will efficiently release the original cytotoxic drug in its active form. Internalization of the ADC commonly proceeds via a clathrin-coated pit mechanism into the endosome-lysosome pathway. ${ }^{17}$ The $\mathrm{pH}$ of these organelles, which varies from $\mathrm{pH}$ 6.0-6.2 (early endosomes), to $\mathrm{pH} 5.5$ (late endosomes), and to $\mathrm{pH} 4.5-5.0$ (lysosomes), plays a critical role in the reaction that transforms the ADC from pro-drug into active drug. ${ }^{18}$ In fact, based on its chemical nature (which will be described in great detail in the following paragraphs), the linker can either be cleaved by the acidic conditions or, if non-cleavable, will be released together with the cytotoxic molecule upon proteolytic degradation of the antibody portion.

\section{Drug action}

Once released, many factors will affect the efficiency of the cytotoxic drug inside cells. Beside the issues already discussed, like the rate of internalization of the conjugate and the rate of degradation of its protein moiety that allows the release of the cytotoxic drug, other processes might occur and must be considered, like the rate of efflux of the drug from the endosomal/lysosomal compartment into the cytoplasm, the rate of its efflux outside the cell, and the rate of conversion of the drug into non-active species, if such a process takes place. ${ }^{19}$ The sum of all these events implies that the active drug will be present in the cell only at very low concentrations and because of that, it must be a very potent drug with subnanomolar half maximal inhibitory concentration.

Early ADCs used clinically approved chemotherapeutic drugs, because they were readily available and their toxicological properties were well known. These, however, were only moderately potent and usually less cytotoxic for the targeted tumor cells than the corresponding unconjugated 
drug. ${ }^{20}$ The interest turned then toward those compounds found to be too toxic when tested as a stand-alone chemotherapy agent. These toxins can be 100 to 1,000 times more cytotoxic than traditional anticancer agents. ${ }^{21}$ The number of cytotoxic drugs that fulfill this potency and stability requirement is quite limited. Among these are 1) inhibitors of tubulin polymerization (like the maytansinoids [DMs], dolastatins, auristatin, and cryptophycin); 2) DNA alkylating agents (CC-1065 analogs and duocarmycin); and 3) the enediyne antibiotics such as calicheamicin and esperamicin, which catalyze DNA double-strand breaks. ${ }^{20}$

\section{ADCs: a new innovation in biopharmaceuticals}

ADCs are at the cutting edge of cancer chemotherapy and represent one of the most advanced stages of antibodies evolution. Considering their relatively recent introduction into the drug domain, the fast transformation pace that they are experiencing is, in fact, surprising. This is related to the fact that antibodies are really starting to maintain their promise to become magic bullets and hence, they exert a fascinating interest on both pharmaceutical companies and scientists.

Because of the way this evolutionary process is taking place, it is very likely that several examples of ADCs, similar to ado-trastuzumab emtansine (Genentech, Inc., South San Francisco, CA, USA), will become available in the near future. This drug has the peculiarity to be the conjugated counterpart of trastuzumab, a previously naked antibody still used in therapy, with the consequent possibility to closely observe the difference in efficacy of an antibody and its conjugated counterpart in the very same patient.

All three ADC building blocks are the object of highly intensive manipulation in order to improve the design of the final molecule. Among them, antibodies are being engineered in new formats, linkers are reaching a new maturity through a second generation of site-specific conjugation techniques, and molecules previously impossible to use for their extreme toxicity are now being conjugated and tested.

\section{Linker technology: design and optimization}

Almost neglected at the beginning of the ADC era, linker design has today become an independent chapter in the ADC pipeline, especially because, along with MAbs and cytotoxic drugs, the type of linker, its stability, site of conjugation, and the final drug/antibody ratio (DAR) are crucial parameters which affect ADC biophysical properties, safety, pharmacokinetics (PK), and efficacy. The junction between the antibody and the payload is generally a covalent bond or a chemical linker, while a fusion construct, including the $\mathrm{MAb}$ fragment and the effector joined by a peptide linker, can be devised in the case of an immunotoxin conjugate molecule; thus, expression of the entire polypeptide can be performed in a host.

Linkers are classified into two categories: cleavable and uncleavable (Figure 3). They need to be stable in the circulation and must be cleaved, ideally, only inside the cells, thus preventing the drug payload from being shed before reaching the tumoral cell target. When the linker is unstable in the extracellular space, the free drug subsequently permeates the cell to reach its target. ${ }^{22}$ This can be a useful mechanism to enhance on-target activity, but it can also be the cause of offtarget toxicity, which is absent with uncleavable linkers. ${ }^{21}$

Uncleavable linkers are cut by complete degradation of the MAb component in the lysosome, which leaves an active unit formed by the drug, the linker, and a lysine residue. ${ }^{23,24}$ Cleavable linkers are selectively cleaved upon change of a biophysical property (eg, $\mathrm{pH}$ in the case of hydrazone linkers), ${ }^{21}$ or when an appropriate protease is found. For example, lysosomal proteases (eg, cathepsin and plasmin) are present at elevated levels in certain tumor tissues. ${ }^{25}$

Though established conjugation chemistries and linkers for antibody arming have already led to a significant improvement in therapeutic outcome, ${ }^{26}$ a rapid evolution of both into a second generation is already under way. The reason is mainly to be found in the heterogeneity of conjugation sites and the number of drug molecules for a given ADC, which has, in fact, been found to condition the activity of the final drug.

Some effort has been put into the generation of reproducible heterogeneity (ado-trastuzumab emtansine) in order to guarantee consistent therapeutic effects. However, a more promising and finer alternative is represented by devising and optimizing new methods for site-specific conjugation, which allow control of location and number of drug moieties per antibody molecule. As antibodies are glycoproteins, the main conjugation strategies target either the primary amino acid sequence or the sugar moieties, exploiting either native structural components or artificial handles introduced by different technologies (eg, bioorthogonal chemistry, tag addition).

\section{Conjugation by amino acid residues}

Conjugation by amino acid residues exploits the thiol group of cysteine or the epsilon amino group of lysine. Cysteinebased strategies already count many variants. Seattle Genetics, Inc. (Bothell, WA, USA) technology exploits four 


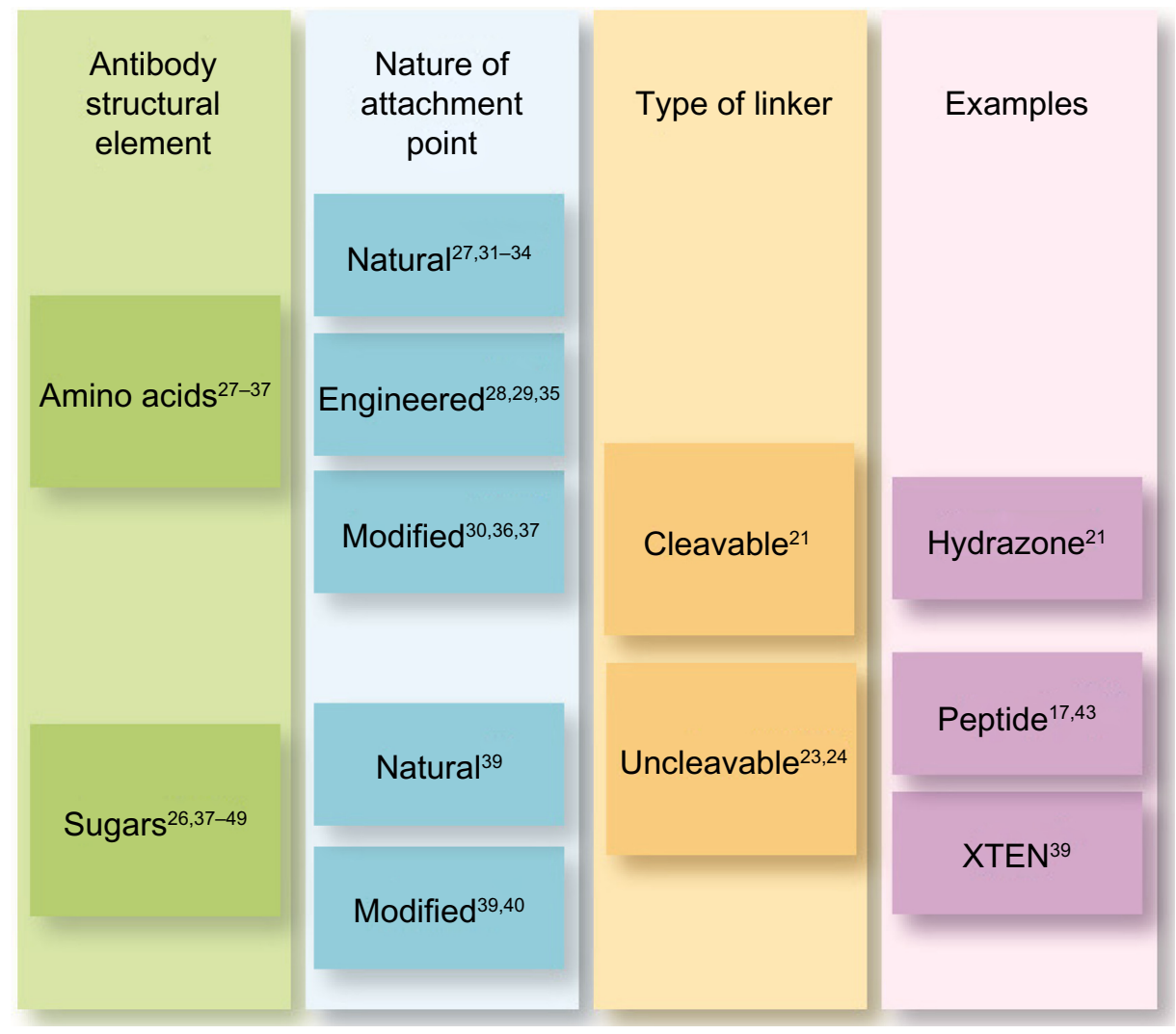

Figure 3 Linkers. Classification of linkers according to the available technologies. Beyond cleavable and uncleavable linkers, site-specific conjugation is the most relevant field of investigation at the moment, with several novel emerging technologies.

Abbreviation: XTEN, unstructured polypeptides that are able to extend the half-life of proteins.

interchain disulphide bridges in the antibody hinge region. Reduction of these four bonds leads to the formation of eight cysteine thiols as linkage points. DAR can be from zero to eight, with the average controlled around four. ${ }^{27}$ ThioMAb drug conjugates are a type of therapeutic antibody to which toxins or chemotherapeutic agents are chemically linked to engineered cysteines, which have been introduced into the amino acid sequence by single point mutations. Typically, two engineered cysteines are introduced per antibody on the heavy chains, light chains, or Fc, which become conjugation sites for linkers. For example, Seattle Genetics, Inc. exploited residue 239 of the antibody heavy chain, generating two unpaired cysteines. ${ }^{28}$ ThioMAb drug conjugates improve the therapeutic index compared to conventional drug conjugation strategies for some antibodies. ${ }^{29}$ Thiobridge ${ }^{\mathrm{TM}}$ conjugation (Polytherics Ltd, London, UK) allows connection of natural cysteines by a three-carbon bridge to which a toxic payload is already conjugated, with a $90 \%$ yield and a DAR of four. ${ }^{27}$ Single or dual selenocysteine residues have also been introduced into the $\mathrm{C}$-terminus of antibodies and allow conjugation without interference with native cysteine bridges ${ }^{30}$ In the case of disulphide bonds, the release of the payload is attributed to the high intracellular concentration of glutathione, as, in the absence of sulfhydryl groups, disulphide bonds are thermodynamically stable.

Lysines are very common in antibody sequences and half of them project their side chain on the surface, ${ }^{31}$ becoming potential conjugation sites. Several ADCs are lysine conjugates ${ }^{31}$ and a recently presented variant of lysine conjugation mediated by light can lead to high drug loads ( $8-10$ drug residues per molecule), ${ }^{32}$ especially if engineered lysine sites are used. In the humanized antibody huC242, lysines were used as attachment points for a bifunctional cross-linking agent then linked to thiol-containing DM1 or DM4. Varying the steric hindrance at carbon atoms adjacent to the disulphide bond led to optimization in in vivo activity. ${ }^{33}$ There are examples where both lysine and cysteine conjugation were tested for the same antibody, with significantly different outcomes. ${ }^{34}$

A glutamine tag can be introduced and exploited for binding, ${ }^{35}$ but an engineered transglutaminase (mTgase) can catalyse direct conjugation of drug molecules using native antibody heavy chains as a substrate (Dophen Biomedical, West Sacramento, CA, USA). Alternatively, a new site-specific conjugation technology based on bacterial 
transglutaminase exploits point mutations in the antibody heavy chain, which generates two or four conjugation sites for enzymes. Linkers have been optimized in order to conjugate to these positions quantitatively. This technology, by Innate Pharma SA (Marseille, France), allows generation of homogeneous ADCs with an exact antibody/toxin ratio of $2: 1$ or 4:1. Non-natural amino acids (otherwise known as unnatural amino acids $)^{36}$ are an alternative adopted by Sutro Biopharma, Inc. (San Francisco, CA, USA) and Ambrx, Inc. (La Jolla, CA, USA), with specifically designed platforms. ${ }^{37}$

\section{Conjugation by sugar residues}

A big chapter in the realm of site-specific conjugation consists of binding through sugar moieties or glycan remodeling. ${ }^{38}$ Glycosylated proteins can now be produced in engineered bacterial hosts carrying glycosylation enzymes. Several chemistries have also been designed for this purpose: metalfree click reactions to label proteins and glycoproteins, posttranslational glycan remodeling, and subsequent metal-free click reaction, plus glycoengineering by aminoxy chemistry. Currently available are also XTEN derivatives containing amino, thiol, and/or dibenzocyclooctyne reactive groups. ${ }^{39}$

Though homogeneity is necessary, finding the best conjugation site is also very relevant because of its effect on the functionality, stability, pharmacology, and toxicology of the resulting ADC. ${ }^{37}$ Among the technologies to engineer new binding sites to the antibody moiety, great interest has arisen for bioorthogonal chemistry. This relatively recent type of chemistry ${ }^{40}$ introduces functional groups into cellbuilt proteins exploiting substrates, which can be processed and included into biological molecules without interfering with their physiological activity, in an "orthogonal" way to normal functional groups. The handles thus generated can then be used for conjugation of the purified protein. Both sugar and non-sugar moieties can be targeted with this technology. Orthogonal conjugation sites allow the maintenance of an unaltered main function on the antibody side (antigen binding).

\section{Novel linkers}

But beyond conjugation sites, the nature of the linker is also relevant. The peptide-like citrulline-valine linker is selectively cleaved by lysosomal proteases, and it has demonstrated high levels of stability in the serum (brentuximab vedotin, Seattle Genetics, Inc.). ${ }^{17,41}$ Novel linkers are also searched that, ideally, cleave at suitable rates to allow the controlled release of drugs. Among the most recently described are: SNAP, a highly bifunctional linker that yields homogeneous ADCs armed with the toxin (Igenica Biotherapeutics, Burlingame, CA, USA); Thiobridge ${ }^{\mathrm{TM}}$ (Polytherics, Ltd), described in the "Conjugation by amino acid residues" section; CL2A; ${ }^{42}$ and SpaceLink (Synthon BV, Nijmegen, the Netherlands). Finally, fusion proteins can take advantage from inteins technology. ${ }^{43}$

Coupling can also be obtained by non-covalent bonds. One recent technology is the dock-and-lock method, which uses the natural binding occurring between adenosine monophosphate-dependent protein kinase and the anchoring domain of A kinase to assemble multiple units in a single active molecule. ${ }^{44}$

For radiolabelled biomolecules, special linkers (bifunctional coupling or chelating agents) are needed, which meet the need to chelate the tracing radiometal. ${ }^{45}$ For diagnostic purposes, bifunctional coupling agents need to be retained in the circulation only for a short time, until their excretion kinetics can be modified with various PKmodifying linkers (cationic, anionic, neutral, or metabolically cleavable) to improve renal clearance or to slow extraction by hepatocytes. ${ }^{45}$

Optimization of an ADC linker is often laborious, and several case studies suggest that a change in linker type can strongly affect the outcome. For example, a disulphide linker with variable hindrance was tested for ado-trastuzumab emtansine, ${ }^{16,46}$ and several conjugates were tested for the same antibody exploiting an aldehyde group. ${ }^{47}$

Linker design and optimization is still a trial and error process, but some clear principles are falling into place. ${ }^{26}$ At the same time, there is a pressing need for more specific analytical techniques, useful to assess the structural effect of conjugation, ${ }^{48}$ and this becomes even more relevant on the manufacturing side of ADCs, where further issues need to be addressed (like, for example, in the case of trisulphide modification). ${ }^{49}$

\section{The ADC pipeline}

Among the ADCs currently in clinical trials for the treatment of different types of cancers, the first ADCs developed and approved by the FDA (gemtuzumab ozogamicin, now discontinued, and brentuximab vedotin) were directed toward hematological malignancies, which are more easily reachable and accessible to drugs. Many other candidates currently in advanced clinical investigation target blood tumors: inotuzumab ozogamicin (Pfizer, Inc.), an anti-CD22 calicheamicin conjugate, is directed toward advanced acute lymphoblastic leukemia (ALL) and is currently in phase III study (INOVATE ALL Study 1022, National Clinical Trial [NCT] number 01564784) for the treatment of this condition, while it has been 
discontinued in non-Hodgkin lymphoma (NHL), as no increased effect was shown compared to existing therapies. ${ }^{50}$ Polatuzumab vedotin (anti-CD79b-MMAE [monomethyl auristatin E]) and pinatuzumab vedotin (anti-CD22-MMAE) are in a phase II trial (ROMULUS, NCT01691898) in combination with rituximab to treat relapsed refractory NHL, with bearable toxicity and a suggested greater activity of polatuzumab vedotin from preliminary results. ${ }^{51}$ However, biotechnology companies are increasingly trying to apply the technology to the treatment of solid tumors as well (Figure 4). A promising boost to this approach came from the FDA approval of ado-trastuzumab emtansine in February 2013 for human epidermal growth factor receptor $2+($ HER2 +$)$ metastatic breast cancer, after failure of trastuzumab plus chemotherapy regimens. Trastuzumab is an anti-HER2 antibody whose linkage to maytansinoid DM1 showed a 3-month increase in progression-free survival and a 6-month increase in overall survival compared to standard therapies in advanced breast cancers, with a concomitant reduction of adverse effects (TH3RESA phase III clinical trial, NCT01419197)..$^{52}$ The drug is also in evaluation as first-line treatment for patients with HER2+ metastatic breast cancer or recurrent locally advanced breast cancer. ${ }^{53}$

Other compounds are also showing promising results in phase II clinical trials: glembatumumab vedotin, or CDX011 (Celldex Therapeutics, Inc., Hampton, NJ, USA), an $\mathrm{ADC}$ directed against the surface glycoprotein nonmetastatic melanoma protein B (GPNMB), showed beneficial activity in patients with triple-negative (estrogen receptor/progesterone receptor/HER2-) breast cancer overexpressing GPNMB ( $\geq 25 \%$ of tumor cells), ${ }^{54}$ and a second phase II clinical trial (METRIC, NCT01997333) started in December 2013 for an accelerated approval of the drug in triple-negative breast cancer overexpressing GPNMB. IMMU-132 and IMMU-130 (Immunogen, Inc., Waltham, MA, USA) showed disease stabilization as assessed by computed tomography in phase II clinical trials in solid tumors (NCT01631552) and metastatic colorectal carcinomas (NCT01605318), respectively. ${ }^{55,56}$

Indatuximab ravtansine (BT062, Biotest AG, Dreieich, Germany) is under investigation in metastatic urinary bladder cancer and metastatic triple-negative breast cancer after positive results obtained in multiple myeloma. ${ }^{57}$ SAR-3419 (Sanofi SA, Paris, France) showed significant activity with an overall response rate of $43.9 \%$, and acceptable side effects in patients with relapsed/refractory diffuse large-cell lymphoma. ${ }^{58}$ Preliminary results from phase I clinical trial for ABT-414 (AbbVie, Inc., Chicago, IL, USA), ${ }^{59}$ an anti-EGFR (epidermal growth factor receptor)/anti-monomethyl auristatin F conjugate for the treatment of glioblastoma multiforme, have shown encouraging efficacy, and the drug is currently in a phase I/II clinical trial in subjects with solid tumours likely to express EGFR (NCT01741327); additionally, the European Medicines Agency (EMA) and FDA granted orphan drug designation to the drug in August 2014 in the therapy of glioblastoma multiforme.$^{60}$ DNIB0600A (anti-sodium-dependent phosphate transport protein $2 \mathrm{~b}-\mathrm{MMAE}$ conjugated by Genentech, Inc.) is in phase II clinical trial in comparison with PEG (polyethylene glycol)-ylated liposomial doxorubicin for platinum-resistant ovarian cancer, primary peritoneal cancer, and fallopian tube cancer (trial number NCT01991210). Several other drugs are currently in phase I clinical trials: we have previously extensively reviewed the building blocks of the main promising $\mathrm{ADCs},{ }^{61}$ and useful and updated information can also be found in the literature. ${ }^{62-65}$ We will instead focus here on areas and novelties, which companies are exploring to create potent, specific, and well-tolerated ADCs.

A first point to consider is the selection of the target. Most of the thus far used targets for ADCs have been represented by receptors of the leukocyte differentiation antigen family,

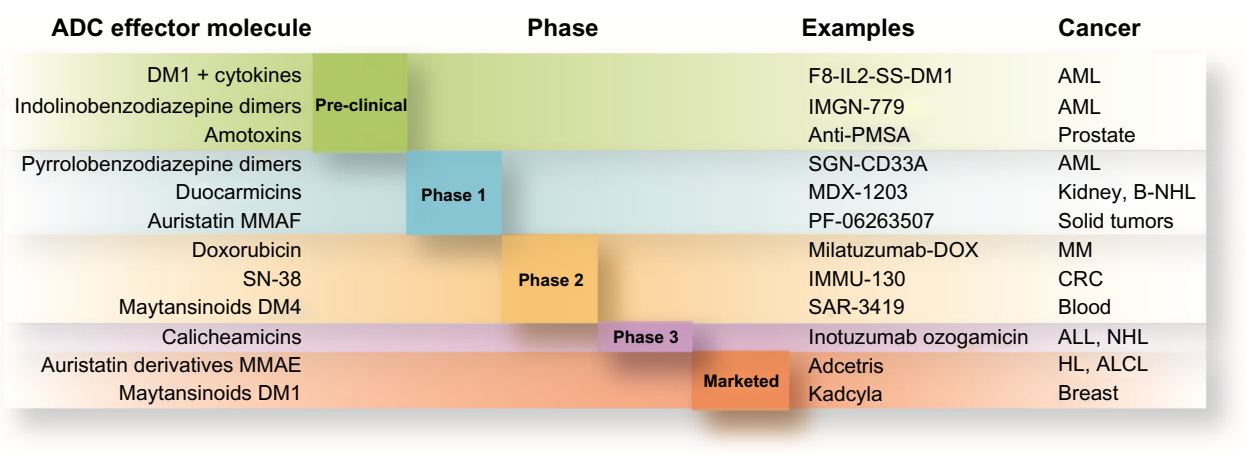

Figure 4 ADC pipeline. Clinical phase of ADCs classified by effectors, and target tumors.

Abbreviations: ADC, antibody-drug conjugate; DM, maytansinoid; MMAF, monomethyl auristatin F; MMAE, monomethyl auristatin E; PSMA, prostate-specific membrane antigen; AML, acute myeloid leukemia; NHL, non-Hodgkin lymphoma; CRC, complement-dependent cytotoxicity; ALL, acute lymphoblastic leukemia, MM, multiple myeloma; HL, Hodgkin lymphoma; ALCL, anaplastic large-cell lymphoma; B-NHL, B type non-Hodgkin lymphoma. 
which undergo rapid internalization after binding to the drug. Some of them, like CD70 (target of Seattle Genetics' SGN-CD70A, see later in this section); CD74 or CD56; and CD117 (target of LOP-628, Novartis International AG, Basel, Switzerland/Immunogen's maytansine-conjugated ADC just entered in phase I clinical trial, NCT02221505) are not only expressed in hematological malignancies, but have also been exploited as targets for solid tumors. Other targets, among which are MUC1, MUC16, EGFR, EphB2, and PMSA (prostate membrane-specific antigen), are tissue-specific molecules targeted in the attempt to improve a selective ADC action on solid tumors. ${ }^{14,62,63}$

On the other hand, companies are exploring molecules over-expressed and activated in many solid tumors, in order to tackle several different types of cancers, especially highly invasive and metastatic cancers, with the same drug. For example, HuMax-TF-ADC (MMAE-conjugated anti-tissue factor antibody by Genmab A/S, Copenhagen, Denmark) has entered a phase I clinical trial in late 2013 in patients with a range of solid tumors (NCT02001623); another agent targeting the widely distributed AXL kinase antigen ${ }^{66}$ should also enter the clinical phase soon after promising preclinical data. ${ }^{67}$ Other strategies aim at targeting molecules involved in tumor immunosurveillance and regulation of tumor inflammatory microenvironment, like TIM-1 (CDX-014, Celldex Therapeutics, Inc.), which is expected to enter a phase I clinical trial for renal cell carcinoma in 2015. ${ }^{68,69}$

Current research in the field is focusing on the identification of criteria that could help to determine in advance proper candidates for targeting, searching for molecules that retain not only suitable expression profiles but also good properties in terms of pharmacodynamics and rates of internalization. The presence of specific sequences in the cytosolic part (tyrosine- or dileucine-based linear motifs), ${ }^{70}$ conformational patches of amino acids on the surface of folded intracellular domains, ${ }^{71}$ or palmitoylation of cysteine residues ${ }^{72}$ may help to predict the rate of internalization.

The influence of co-receptors should also be considered. ${ }^{73,74}$ Even if a rapid internalization is generally desired, the penetration of the drug, especially in the context of some solid tumors, could be hindered by an excessive turnover, leading to target downregulation at the cell surface. Thus, mathematical models useful to predict the contribution of antigen properties to tumor penetration and efficacy of the drug have been developed and could help, in some cases, to drive the choice toward slowly internalizing targets. ${ }^{75}$

Combining traditional chemotherapy and ADCs has also resulted in targeting proteins normally not expressed on the cell surface, but induced instead either by chemotherapy ${ }^{76}$ or exposed after loss of membrane integrity due to previous chemotherapy-induced cell death. The latter is the case, for instance, of the La autoantigen, selectively induced in dead cancer cells after DNA-damaging cisplatin therapy. The selective binding of the drug to this target could improve the efficacy of other treatments, such as radioimmunotherapy, against the residual tumor mass. ${ }^{77}$ Thanks to the easy accessibility, targeting of vascular ligands has also been started, with encouraging results. ${ }^{78,79}$

Recent findings suggest that non-internalizing ADCs could also exert therapeutic activity. ${ }^{80}$ In parallel, an appropriate selection and stratification of the target population of patients is fundamental for a successful therapeutic strategy. The gold standard remains immunohistochemistry, especially in combination with tissue microarray techniques and image

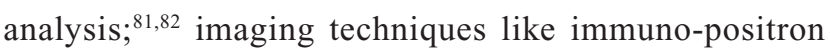
emission tomography are gaining increasing importance in defining ADC potency and safety. ${ }^{83}$

Mathematical modeling could also be useful to predict the PK and absorption, distribution, metabolism, and excretion of potential candidates that are ready to be taken from preclinical to clinical stage. Several models have been developed for the different stages of drug characterization. These models predict systemic distribution of the drug, from the cellular level to tumor, and can define the best drug-to-antibody ratio for an individual ADC. ${ }^{84-86}$

Concerning payloads, as already noted, most of the drugs currently in clinical trials are represented by microtubule assembly inhibitors, both in the form of synthetic auristatin derivatives, or maytansinoid-based ADCs; a minor part is formed by calicheamicin or duocarmycin analogs, which bind to DNA. ${ }^{87}$ The most important pitfall in using tubulin binders is due to the fact that their action is exerted mainly on proliferating cells; in addition, the hydrophobicity, in particular of the maytansinoid-based molecules, could increase the toxicity of the compound. Calicheamicin analogs are hindered by a narrow therapeutic window. Several approaches have been started to face these problems. Immunogen has developed a new class of cytotoxic agents, IGN or indolinobenzodiazepine dimers, which exert an alkylating effect on DNA, arresting the cells in the G2/M phase of the cell cycle in a dose-dependent manner. One of these, DGN-462, has shown a high activity on mice AML xenografts in combination with anti-CD33 antibody. ${ }^{88}$ The related investigational new drug (IND) application for this drug, IMG-779, is foreseen in 2015 for the treatment of relapsing AML. ${ }^{89}$ Pyrrolobenzodiazepine dimers are also 
being explored to confer increased selectivity and potency to the DNA alkylating action. For example, Seattle Genetics' SGN-CD33A (NCT01902329) and SGN-CD70A (NCT02216890) are currently in phase I clinical trials for the treatment of AML, and NHL and renal cell cancer, respectively (as previously noted, CD70 is expressed both in hematological and in solid tumours). IND filing for ADCT401 (ADC Therapeutics Sarl, Lausanne, Switzerland), a PDB-conjugate anti-PMSA drug, is expected in 2015, focusing on hormone-refractory prostate cancer. ${ }^{90-92}$

Conjugation with amotoxin cyclopeptides derived from mushrooms of the Amanita genus, which target RNA synthesis by interaction with RNA polymerase II, represents another option. Their strong hydrophilic nature facilitates coupling reactions thanks to high solubility, reduces the probability of aggregation, and promotes a fast clearance of the molecule. ${ }^{93}$ An anti-PMSA antibody developed by Heidelberg Pharma $\mathrm{GmbH}$ (Ladenburg, Germany) showed high preclinical antitumor activity. ${ }^{94} \mathrm{SN}-38$, the active metabolite of the topoisomerase-I inhibitor irinotecan (CPT-11) has been conjugated with labetuzumab, a mildly reduced, anti- carcinoembryonic antigen-related cell adhesion molecule 5 (CEACAM5) humanized MAb, and the resulting ADC (IMMU-130) is undergoing Phase I and II clinical trials, as already noted in this section. ${ }^{55}$

An additional very promising approach comes from the conjugation of a cytokine, a drug, and an antibody in the same multi-payload molecule: the combination of these moieties as single agents has already shown preclinical efficacy, but the translation to the clinics is progressing slowly due to the need for studying each molecule's pharmacological profile individually before considering combinations on patients. Linking all the components in a single drug could significantly optimize efficacy; Philochem AG (Otelfingen, Switzerland) has very recently released data demonstrating the efficacy of F8-IL2 (interleukin 2)-SS-DM1 on AML preclinical tumor models: F8-IL2-SS-DM1 is a drug combining the immune-stimulating properties of IL2 and the toxic activity of DM1 on the same anti-fibronectin diabody scaffold, and represents the first candidate of a new "immunocytokine-drug conjugate" class of drugs. ${ }^{95}$ A visual summary of the ADC payload pipeline is provided in Figure 4, where the representatives of the different classes, which are in the most advanced clinical trial phase, are listed as examples.

The current status and the research and development approaches attempted in the linker design area have already been explored in the previous "Novel linkers" section. Thanks to the previously mentioned SpaceLink technology and the optimized biological activity that this linker chemistry could determine, particularly in combination with specific duocarmycin derivatives (refer to the "Novel linkers" section), Synthon's anti-HER2 ADC SYD985 has shown superior antitumor activity in a preclinical head-to-head comparison study with ado-trastuzumab emtansine in gastric and breast cancers, ${ }^{96}$ including low-HER2 expressing breast cancers, and has entered the clinical phase by the end of 2014 (NCT02277717). ${ }^{97}$

\section{Future directions}

Despite the great development of ADC technology during the last two decades, mirrored and propelled by a growing increase in interest and investments in the field both by industries and by the research community, a lot of work is still needed to approach ADCs with characteristics really tailored to the specific components and the specific type of cancer targeted by each drug.

The most important contribution to this goal could probably be derived from the engineering of the carrier moiety of the ADC. This could be intended as further analysis of the canonical full-length antibody format, understanding its toxicity and therapeutic index profile, and as the substitution of the antibody format with other formats with more desirable features, known as "alternative scaffolds". Most of the antibodies currently in clinical trials are full-length MAbs of the immunoglobulin (Ig) G1 isotype, either chimeric, humanized, or fully human. ${ }^{64}$ Through their Fc portion, they stimulate effector functions like antibody-dependent cell-mediated cytotoxicity, phagocytosis, and complementdependent cytotoxicity. As the activation of these patterns could increase the overall toxicity of the drug, IgG2 and IgG4, whose Fc portions have a reduced affinity for their receptor and could thus limit effector functions, have been tested as vehicles for drugs (eg, of gentuzumab ozogamicin). However, contrasting evidence exists: no differences in ADC function depending on the format have been demonstrated so far. ${ }^{98}$ Nonetheless, a systematic analysis has not yet been performed. A recent advance in this perspective has come from the POTELLIGENT technology (BioWa, Inc., Princeton, NJ, USA), which consists in the elimination of fucose from sugar chains of antibodies, determining a significant increase in antibody-dependent cell-mediated cytotoxicity and, consequently, in the efficacy of the ADC. Oxford BioTherapeutics, Inc. (San Jose, CA, USA) has applied this technology to its OBT357/MEN1112 drug, which has shown promising in vitro efficacy and has entered Phase I clinical trial in late 2014 for the treatment of advanced AML. ${ }^{99}$

Alternative scaffolds are small polypeptide frameworks designed on Ig backbones (nanobodies, domain antibodies, 
bifunctional scaffolds) or deriving from completely unrelated proteins exploited for their high solubility and stability compared to full-length antibodies. ${ }^{100}$ While the first molecules developed in this class showed very poor efficacy due to their low molecular weight and subsequent fast clearance from the organism, several strategies, from PEGylation to more elaborate approaches such as polysialylation, amino acid polymers, and albumin-binding derivatives have been attempted successfully to overcome this difficulty. ${ }^{101}$ Thanks to their reduced toxicity and PK that are easier to predict and control, they can provide a great help in improving specificity and in improving control of biodistribution and clearance in the ADC field.

In close relation, improvement in understanding ADCs' $\mathrm{PK}$ and pharmacodynamics is mandatory for optimal design of new candidates. In particular, appropriate models to integrate the assessed pharmacological properties of each component of the complex drug have to be optimized, together with a deeper characterization of the fate and interactions of the catabolytes deriving from each moiety. ${ }^{102}$

One further current limitation of ADCs is linked to the development of multi-drug resistance to the cytotoxic payloads. The process is caused by transporters of the adenosine triphosphate-binding cassette family, among which in particular P-glycoprotein (MDR1) plays a central role, promoting an active efflux of the internalized drug outside the cell. ${ }^{103}$ Modification of the toxin or of the linker ${ }^{19}$ and PEG coupling ${ }^{104}$ have been attempted to generate export-resistant ADCs, but the results have not been satisfying because of the appearance of other unknown resistance mechanisms.

Finally, while almost every therapeutic developed so far in the field has made use of the endolysosome-mediated route of internalization, alternative pathways to the endoplasmatic reticulum and trans-Golgi network could be exploited to overcome potential pharmacodynamic difficulties related to the hydrolytic environment of the former pathway. ${ }^{105}$

\section{Acknowledgments}

LI is supported by a fellowship from the University of Pavia; CS' salary comes from Ministry for Education, University and Research; LV has a European Fellowship from the School of Specialization in Clinical Pathology.

\section{Disclosure}

The authors report no conflicts of interest in this work.

\section{References}

1. Chabner BA, Roberts TG Jr. Timeline: chemotherapy and the war on cancer. Nat Rev Cancer. 2005;5(1):65-72.
2. Köhler G, Milstein C. Continuous cultures of fused cells secreting antibody of predefined specificity. Nature. 1975;256(5517): 495-7.

3. Scott AM, Wolchok JD, Old LJ. Antibody therapy of cancer. Nat Rev Cancer. 2012;12(4):278-287.

4. Wu AM, Senter PD. Arming antibodies: prospects and challenges for immunoconjugates. Nat Biotechnol. 2005;23(9):1137-1146.

5. Nelson AL, Dhimolea E, Reichert JM. Development trends for human monoclonal antibody therapeutics. Nat Rev Drug Discov. 2010;9(10):767-774.

6. Kreitman RJ. Toxin-labeled monoclonal antibodies. Curr Pharm Biotechnol. 2001;2(4):313-325.

7. Sharkey RM, Goldenberg DM. Perspectives on cancer therapy with radiolabeled monoclonal antibodies. $J$ Nucl Med. 2005;46(Suppl 1): 115S-127S.

8. Payne G. Progress in immunoconjugate cancer therapeutics. Cancer Cell. 2003;3(3):207-212.

9. Evans JB, Syed BA. From the analyst's couch: next-generation antibodies. Nat Rev Drug Discov. 2014;13(6):413-414.

10. Bross PF, Beitz J, Chen G, et al. Approval summary: gemtuzumab ozogamicin in relapsed acute myeloid leukemia. Clin Cancer Res. 2001;7(6):1490-1496.

11. Beck A, Haeuw JF, Wurch T, Goetsch L, Bailly C, Corvaïa N. The next generation of antibody-drug conjugates comes of age. Discov Med. 2010;10(53):329-339.

12. Beck A, Reichert JM. Antibody-drug conjugates: present and future. MAbs. 2014;6(1):15-17.

13. Carter PJ, Senter PD. Antibody-drug conjugates for cancer therapy. Cancer J. 2008;14(3):154-169.

14. Teicher BA. Antibody-drug conjugate targets. Curr Cancer Drug Targets. 2009;9(8):982-1004.

15. Dijoseph JF, Dougher MM, Armellino DC, et al. CD20-specific antibody-targeted chemotherapy of non-Hodgkin's B-cell lymphoma using calicheamicin-conjugated rituximab. Cancer Immunol Immunother. 2007;56(7):1107-1117.

16. Lewis Phillips GD, Li G, Dugger DL, et al. Targeting HER2-positive breast cancer with trastuzumab-DM1, an antibody-cytotoxic drug conjugate. Cancer Res. 2008;68(22):9280-9290.

17. Sutherland MS, Sanderson RJ, Gordon KA, et al. Lysosomal trafficking and cysteine protease metabolism confer target-specific cytotoxicity by peptide-linked anti-CD30-auristatin conjugates. J Biol Chem. 2006;281(15):10540-10547.

18. Weisz OA. Acidification and protein traffic. Int Rev Cytol. 2003;226: 259-319.

19. Kovtun YV, Goldmacher VS. Cell killing by antibody-drug conjugates. Cancer Lett. 2007;255(2):232-240.

20. Lambert JM. Drug-conjugated monoclonal antibodies for the treatment of cancer. Curr Opin Pharmacol. 2005;5(5):543-549.

21. Ducry L, Stump B. Antibody-drug conjugates: linking cytotoxic payloads to monoclonal antibodies. Bioconjug Chem. 2010;21(1): 5-13.

22. Polson AG, Calemine-Fenaux J, Chan P, et al. Antibody-drug conjugates for the treatment of non-Hodgkin's lymphoma: target and linker-drug selection. Cancer Res. 2009;69(6):2358-2364.

23. Erickson HK, Park PU, Widdison WC, et al. Antibody-maytansinoid conjugates are activated in targeted cancer cells by lysosomal degradation and linker-dependent intracellular processing. Cancer Res. 2006;66(8):4426-4433.

24. Chari RV. Targeted cancer therapy: conferring specificity to cytotoxic drugs. Acc Chem Res. 2008;41(1):98-107.

25. Koblinski JE, Ahram M, Sloane BF. Unraveling the role of proteases in cancer. Clin Chim Acta. 2000;291(2):113-135.

26. Flygare JA, Pillow TH, Aristoff P. Antibody-drug conjugates for the treatment of cancer. Chem Biol Drug Des. 2013;81(1):113-121.

27. Thayer Ann M. Site specific: developers aim to create well-defined drug conjugates. Chem Engineer News. 2014;92(3):13-21. 
28. Django S, Lindsay T, Lori W, et al. Abstract 4634: Engineered cysteine drug conjugates show potency and improved safety. Cancer Res. 2012; 72(8 Suppl 1).

29. Junutula JR, Raab H, Clark S, et al. Site-specific conjugation of a cytotoxic drug to an antibody improves the therapeutic index. Nat Biotechnol. 2008;26(8):925-932.

30. Patterson JT, Asano S, Li X, Rader C, Barbas CF, 3rd. Improving the serum stability of site-specific antibody conjugates with sulfone linkers. Bioconjug Chem. Epub August 6, 2014.

31. Feng Y, Zhu Z, Chen W, Prabakaran P, Lin K, Dimitrov DS. Conjugates of small molecule drugs with antibodies and other proteins. Biomedicines. 2014;2:1-13.

32. Photobiotics [homepage on the Internet]. Innovative targeted therapeutics from photobiotics. Available from: http://www.photobiotics.com technology/targeting-platform-optilink.shtml. Accessed October 28, 2014.

33. Kellogg BA, Garrett L, Kovtun Y, et al. Disulfide-linked antibodymaytansinoid conjugates: optimization of in vivo activity by varying the steric hindrance at carbon atoms adjacent to the disulfide linkage. Bioconjug Chem. 2011;22(4):717-727.

34. Wilbur DS, Chyan MK, Nakamae H, et al. Reagents for astatination of biomolecules. 6. An intact antibody conjugated with a maleimidocloso-decaborate(2-) reagent via sulfhydryl groups had considerably higher kidney concentrations than the same antibody conjugated with an isothiocyanato-closo-decaborate(2-) reagent via lysine amines. Bioconjug Chem. 2012;23(3):409-420.

35. Panowksi S, Bhakta S, Raab H, Polakis P, Junutula JR. Site-specific antibody drug conjugates for cancer therapy. MAbs. 2014;6(1): 34-45.

36. Hallam TJ, Smider VV. Unnatural amino acids in novel antibody conjugates. Future Med Chem. 2014;6(11):1309-1324.

37. Strop P, Liu SH, Dorywalska M, et al. Location matters: site of conjugation modulates stability and pharmacokinetics of antibody drug conjugates. Chem Biol. 2013;20(2):161-167.

38. Okeley NM, Toki BE, Zhang X, et al. Metabolic engineering of monoclonal antibody carbohydrates for antibody-drug conjugation. Bioconjug Chem. 2013;24(10):1650-1655.

39. Schellenberger V, Wang CW, Geething NC, et al. A recombinant polypeptide extends the in vivo half-life of peptides and proteins in a tunable manner. Nat Biotechnol. 2009;27(12):1186-1190.

40. Hang HC, Yu C, Kato DL, Bertozzi CR. A metabolic labeling approach toward proteomic analysis of mucin-type O-linked glycosylation. Proc Natl Acad Sci U S A. 2003;100(25):14846-14851.

41. Doronina SO, Toki BE, Torgov MY, et al. Development of potent monoclonal antibody auristatin conjugates for cancer therapy. Nat Biotechnol. 2003;21(7):778-784.

42. Immunomedics [homepage on the Internet]. ADC linker. Available from: http://www.immunomedics.com/linker-demo.shtml. Accessed October 28, 2014.

43. Möhlmann S, Bringmann P, Greven S, Harrenga A. Site-specific modification of ED-B-targeting antibody using intein-fusion technology. BMC Biotechnol. 2011;11:76.

44. Chang CH., Rossi EA, Goldenberg DM. The dock and lock method: a novel platform technology for building multivalent, multifunctional structures of defined composition with retained bioactivity. Clin Cancer Res. 2007;13(18 Pt 2):5586s-5591s.

45. Liu S. Bifunctional coupling agents for radiolabeling of biomolecules and target-specific delivery of metallic radionuclides. Adv Drug Deliv Rev. 2008;60(12):1347-1370.

46. LoRusso PM, Weiss D, Guardino E, Girish S, Sliwkowski MX. Trastuzumab emtansine: a unique antibody-drug conjugate in development for human epidermal growth factor receptor 2-positive cancer. Clin Cancer Res. 2011;17(20):6437-6447.

47. Albers AE, Garofalo AW, Drake PM, et al. Exploring the effects of linker composition on site-specifically modified antibody-drug conjugates. Eur J Med Chem. Epub August 23, 2014.
48. Pan LY, Salas-Solano O, Valliere-Douglass JF. Conformation and dynamics of interchain cysteine-linked antibody-drug conjugates as revealed by hydrogen/deuterium exchange mass spectrometry. Anal Chem. 2014;86(5):2657-2664.

49. Cumnock K, Tully T, Cornell C, et al. Trisulfide modification impacts the reduction step in antibody-drug conjugation process. Bioconjug Chem. 2013;24(7):1154-1160.

50. Dang NH, Ogura M, Castaigne S, et al. Randomized, phase 3 trial of inotuzumab ozogamicin plus rituximab (R-InO) versus chemotherapy for relapsed/refractory aggressive B-cell non-Hodgkin lymphoma (B-NHL). Paper presented at: 2014 Annual Meeting of the American Society of Clinical Oncology; Chicago, IL.

51. Morschhauser F, Flinn I, Advani RH, et al. Preliminary results of a phase II randomized study (ROMULUS) of polatuzumab vedotin (PoV) or pinatuzumab vedotin (PiV) plus rituximab (RTX) in patients (Pts) with relapsed/refractory (R/R) non-Hodgkin lymphoma (NHL). Paper presented at: 2014 Annual Meeting of the American Society of Clinical Oncology; Chicago, IL.

52. Wildiers H, Kim SB, Gonzalez-Martin A, et al. T-DM1 for HER2positive metastatic breast cancer $(\mathrm{MBC})$ : primary results from TH3RESA, a phase 3 study of T-DM1 vs treatment of physician's choice. Paper presented at: European Cancer Congress 2013; Amsterdam, the Netherlands.

53. Hurvitz SA, Dirix L, Kocsis J, et al. Phase II randomized study of trastuzumab emtansine versus trastuzumab plus docetaxel in patients with human epidermal growth factor receptor 2-positive metastatic breast cancer. J Clin Oncol. 2013;31(9):1157-1163.

54. Yardley DA, Melisko ME, Forero A, et al. Abstract OT2-6-16: A pivotal multicenter, randomized, study evaluating the novel antibodydrug conjugate CDX-011 in patients with metastatic, triple-negative, high GPNMB over-expressing breast cancer. Paper presented at: San Antonio Breast Cancer Symposium 2013; San Antonio, TX

55. Dotan E, Berlin J, Starodub A, et al. Activity of IMMU-130 antiCEACAM5-SN-38 antibody-drug conjugate (ADC) on metastatic colorectal cancer $(\mathrm{mCRC})$ having relapsed after CPT-11: Phase I study. Paper presented at: 2014 Annual Meeting of the American Society of Clinical Oncology; Chicago, IL.

56. Starodub A, Ocean AJ, Guarino MJ, et al. IMMU-132, an SN-38 antibody-drug conjugate (ADC) targeting Trop-2, as a novel platform for the therapy of diverse metastatic solid cancers: clinical results. Paper presented at: 2014 Annual Meeting of the American Society of Clinical Oncology; Chicago, IL.

57. Kelly KR, Chanan-Khan A, Somlo G, et al. Indatuximab ravtansine (BT062) in combination with lenalidomide and low-dose dexamethasone in patients with relapsed and/or refractory multiple myeloma: clinical activity in len/dex-refractory patients. Paper presented at: 55th American Society of Hematology Annual Meeting 2013; San Francisco, CA.

58. Trneny M, Verhoef G, Dyer MJS, et al. Starlyte phase II study of coltuximab ravtansine (CoR, SAR3419) single agent: Clinical activity and safety in patients (pts) with relapsed/refractory (R/R) diffuse large B-cell lymphoma (DLBCL; NCT01472887). Paper presented at: 2014 Annual Meeting of the American Society of Clinical Oncology; Chicago, IL.

59. Gan HK, Fichtel L, Lassman AB, et al. A phase 1 study evaluating ABT-414 in combination with temozolomide (TMZ) for subjects with recurrent or unresectable glioblastoma (GBM). Paper presented at: 2014 Annual Meeting of the American Society of Clinical Oncology; Chicago, IL.

60. Abbvie [homepage on the Internet]. Abbvie receives EMA and FDA orphan drug designation for investigational compound ABT-414 in the treatment of glioblastoma multiforme. Available from: http://abbvie. mediaroom.com/2014-08-04-AbbVie-Receives-EMA-and-FDA-OrphanDrug-Designation-for-Investigational-Compound-ABT-414-in-theTreatment-of-Glioblastoma-Multiforme. Accessed October 28, 2014.

61. Pasquetto MV, Vecchia L, Covini D, Digilio R, Scotti C. Targeted drug delivery using immunoconjugates: principles and applications. J Immunother. 2011;34(9):611-628. 
62. Newman DJ, Cragg GM. Marine-sourced anti-cancer and cancer pain control agents in clinical and late preclinical development. Mar Drugs. 2014;12(1):255-278.

63. Bouchard H, Viskov C, Garcia-Echeverria C. Antibody-drug conjugatesa new wave of cancer drugs. BMCL Digest. 2014;24(23):5357-5363.

64. Sassoon I, Blanc V. Antibody-drug conjugate (ADC) clinical pipeline: a review. Methods Mol Biol. 2013;1045:1-27.

65. Perez HL, Cardarelli PM, Deshpande S, et al. Antibody-drug conjugates: current status and future directions. Drug Discov Today. 2014;19(7): 869-881.

66. Wu X, Liu X, Koul S, Lee CY, Zhang Z, Halmos B. AXL kinase as a novel target for cancer therapy. Oncotarget. 2014;50(20): 9546-9563.

67. Genmab [homepage on the Internet]. Seattle Genetics and Genmab enter into new antibody-drug conjugate collaboration. Available from: http://ir.genmab.com/releasedetail.cfm?ReleaseID=870102. Accessed October 28, 2014

68. Baghdadi M, Jinushi M. The impact of the TIM gene family on tumor immunity and immunosuppression. Cell Mol Immunol. 2014;11(1):41-48.

69. Celldex Therapeutics [homepage on the Internet]. Celldex Therapeutics establishes preclinical proof of concept for new antibody drug conjugate CDX-014. Data presented at the American Association for Cancer Research Conference 2014. Available from: http://ir.celldex.com/ releasedetail.cfm?ReleaseID=838642. Accessed October 28, 2014.

70. Bonifacino JS, Traub LM. Signals for sorting of transmembrane proteins to endosomes and lysosomes. Annu Rev Biochem. 2003;72: 395-447.

71. Traub LM, Bonifacino JS. Cargo recognition in clathrin-mediated endocytosis. Cold Spring Harb Perspect Biol. 2013;5(11):a016790.

72. Schweizer A, Kornfeld S, Rohrer J. Cysteine 34 of the cytoplasmic tail of the cation-dependent mannose 6-phosphate receptor is reversibly palmitoylated and required for normal trafficking and lysosomal enzyme sorting. J Cell Biol. 1996;132(4):577-584.

73. Tedder TF, Inaoki M, Sato S. The CD19-CD21 complex regulates signal transduction thresholds governing humoral immunity and autoimmunity. Immunity. 1997;6(2):107-118.

74. Ingle GS, Chan P, Elliott JM, et al. High CD21 expression inhibits internalization of anti-CD19 antibodies and cytotoxicity of an antiCD19-drug conjugate. Br J Haematol. 2008;140(1):46-58.

75. Ackerman ME, Pawlowski D, Wittrup KD. Effect of antigen turnover rate and expression level on antibody penetration into tumor spheroids. Mol Cancer Ther. 2008;7(7):2233-2240.

76. Rubinfeld B, Upadhyay A, Clark SL, et al. Identification and immunotherapeutic targeting of antigens induced by chemotherapy. Nat Biotechnol. 2006;24(2):205-209.

77. Al-Ejeh F, Darby JM, Brown MP. The La autoantigen is a malignancyassociated cell death target that is induced by DNA-damaging drugs. Clin Cancer Res. 2007;13(18 Pt 2):5509s-5518s.

78. Gerber H.P, Senter PD, Grewal IS. Antibody drug-conjugates targeting the tumor vasculature: current and future developments. MAbs. 2009;1(3):247-253.

79. Prokopiou EM, Ryder SA, Walsh JJ. Tumour vasculature targeting agents in hybrid/conjugate drugs. Angiogenesis. 2013;16(3):503-524.

80. Perrino E, Steiner M, Krall N, et al. Curative properties of noninternalizing antibody-drug conjugates based on maytansinoids. Cancer Res. 2014;74(9):2569-2578.

81. Sauter G, Simon R, Hillan K. Tissue microarrays in drug discovery. Nat Rev Drug Discov. 2003;2(12):962-972.

82. Krueger JS, Lange H, Potts S, Young D. Assessing factors predictive of response to $\mathrm{ADCs}$ for companion diagnostic strategies. Paper presented at: AACR-NCI-EORTC International Conference: Molecular Targets and Cancer Therapeutics 2013; Boston, MA.

83. Klinguer-Hamour C, Strop P Shah DK, Ducry L, Xu A, Beck A. World Antibody-Drug Conjugate Summit, October 15-16, 2013, San Francisco, CA. MAbs. 2014;6(1):18-29.
84. Shah DK, Haddish-Berhane N, Betts A. Bench to bedside translation of antibody drug conjugates using a multiscale mechanistic PK/PD model: a case study with brentuximab-vedotin. J Pharmacokinet Pharmacodyn. 2012;39(6):643-659.

85. Shah DK, Betts AM. Towards a platform PBPK model to characterize the plasma and tissue disposition of monoclonal antibodies in preclinical species and human. J Pharmacokinet Pharmacodyn. 2012;39(1): 67-86.

86. Chudasama VL, Schaedeli Stark F, Harrold JM, et al. Semi-mechanistic population pharmacokinetic model of multivalent trastuzumab emtansine in patients with metastatic breast cancer. Clin Pharmacol Ther. 2012;92(4):520-527.

87. Anderl J, Faulstich H, Hechler T, Kulke M. Antibody-drug conjugate payloads. Methods Mol Biol. 2013;1045:51-70.

88. Whiteman K, Audette C, Dandeneau A, et al. Abstract 2644: Antibodydrug conjugates (ADCs) with a novel DNA-alkylating agent, DGN462, are highly potent in vitro and in vivo against human cancer models. Paper presented at: 105th Annual Meeting of the American Association for Cancer Research 2014; San Diego, CA.

89. Immunogen [homepage on the Internet]. ImmunoGen, Inc. announces preclinical findings for IMGN779, a potential treatment for acute myeloid leukemia. Available from: http://investor.immunogen.com/ releasedetail.cfm?ReleaseID=854832. Accessed October 28, 2014.

90. Hartley JA. The development of pyrrolobenzodiazepines as antitumour agents. Expert Opin Investig Drugs. 2011;20(6):733-744.

91. Kung Sutherland MS, Walter RB, Jeffrey SC, et al. SGN-CD33A: a novel CD33-targeting antibody-drug conjugate using a pyrrolobenzodiazepine dimer is active in models of drug-resistant AML. Blood. 2013;122(8):1455-1463.

92. ADC Therapeutics [homepage on the Internet]. ADC Therapeutics to move antibody drug conjugate ADCT-401 for prostate cancer into human clinical trials with partner MedImmune. Available from: http:// www.adctherapeutics.com/news/2014/05/adc-therapeutics-to-moveantibody-drug-conjugate-adct-401-for-prostate-cancer-into-humanclinical-trials-with-partner-medimmune. Accessed October 28, 2014.

93. Anderl J, Müller C, Heckl-Östreicher B, Wehr R. Abstract 3616: Highly potent antibody-amanitin conjugates cause tumor-selective apoptosis. Paper presented at: 102nd Annual Meeting of the American Association for Cancer Research 2011; Orlando, FL.

94. Hechler T, Kulke M, Mueller C, Pahl A, Anderl J. Abstract 664: Amanitin-based antibody-drug conjugates targeting the prostatespecific membrane antigen. Paper presented at: 105th Annual Meeting of the American Association for Cancer Research 2014; San Diego, CA.

95. List T., Casi G., Neri D. A chemically defined trifunctional antibodycytokine-drug conjugate with potent antitumor activity. Mol Cancer Ther. 2014;13(11):2641-2652.

96. Verheijden G, Beusker P, Ubink R, et al. Toward clinical development of SYD985, a novel HER2-targeting antibody-drug conjugate (ADC). Paper presented at: 2014 ASCO Annual Meeting2014; Chicago, IL.

97. Synthon Biopharmaceuticals [homepage on the Internet]. Synthon's Anti-HER2 ADC frontrunner SYD985 outperforms only available HER2-targeting ADC. Available from: http://www.synthon. com/Corporate/News/PressReleases/Synthons-Anti-HER2-ADCFrontrunner-SYD985-Outperforms-Only-Available-HER2-targetingADC?sc_lang=en. Accessed October 28, 2014.

98. McDonagh CF, Kim KM, Turcott E, et al. Engineered anti-CD70 antibody-drug conjugate with increased therapeutic index. Mol Cancer Ther. 2008;7(9):2913-2923.

99. Oxford BioTherapeutics [homepage on the Internet]. Oxford BioTherapeutics lead programs. Available from: http://www. oxfordbiotherapeutics.com/wp/wp-content/uploads/2014/11/ OBT_Menarini_357_PhI_Enrollment-20141125.pdf. Accessed December 15, 2014.

100. Wurch T, Pierré A. Depil S. Novel protein scaffolds as emerging therapeutic proteins: from discovery to clinical proof-of-concept. Trends Biotechnol. 2012;30(11):575-582. 
101. Constantinou A, Chen C, Deonarain MP. Modulating the pharmacokinetics of therapeutic antibodies. Biotechnol Lett. 2010;32(5): 609-622.

102. Lin K, Tibbitts J. Pharmacokinetic considerations for antibody drug conjugates. Pharm Res. 2012;29(9):2354-2366.

103. Cianfriglia M. Targeting MDR1-P-glycoprotein (MDR1-Pgp) in immunochemotherapy of acute myeloid leukemia (AML). Ann Ist Super Sanita. 2013;49(2):190-208.
104. Kovtun YV, Audette CA, Mayo MF, et al. Antibody-maytansinoid conjugates designed to bypass multidrug resistance. Cancer Res. 2010;70(6):2528-2537.

105. Tarragó-Trani MT, Storrie B. Alternate routes for drug delivery to the cell interior: pathways to the Golgi apparatus and endoplasmic reticulum. Adv Drug Deliv Rev. 2007;59(8):782-797.

Antibody Technology Journal

\section{Publish your work in this journal}

Antibody Technology Journal is international, peer-reviewed, open access journal publishing original research, reports, reviews and commentaries on all areas of antibody technology. The manuscript management system is completely online and includes a very quick and fair

\section{Dovepress}

peer-review system. Visit http://www.dovepress.com/testimonials.php to read real quotes from published authors. 